\title{
Effect of different pore-forming agents on the performance of lightweight external wall brick
}

\author{
Sun Shibing ${ }^{1,}$, Zhang Suli, b ${ }^{1,}$ Li Xin $^{2}$, and Chen Hua ${ }^{1}$ \\ ${ }^{1}$ Beijing University of Technology, Beijing 100124 \\ ${ }^{2}$ China Building Materials Academy, Beijing 100024 \\ a sun8203@vip.sohu.com, ${ }^{b}$ zhangsuli1029@163.com
}

\begin{abstract}
Keywords: Pore-forming agents; Lightweight external wall brick; Bulk density; Water absorption; Flexural strength

Abstract: In this paper, the lightweight external wall brick was prepared by means of dry pressure molding sintering process to explore the effect of the pore-forming agent on its performance. The shale and clay were used as the main raw materials and additives of bran, coal powder and SiC were chosen as pore-forming agent. The results show that, for both different pore-forming agents, as the content of pore-forming increases, the water absorption got elevated and the bulk density and flexural strength got reduced. Moreover, $\mathrm{SiC}$ could react with oxygen to generate $\mathrm{CO}$ and $\mathrm{CO}_{2}$, resulting in the appearance of numerous closed pores inside the green body after fired. It was easy to obtain low density lightweight external wall brick which could exhibit better performance.
\end{abstract}

\section{Introduction}

The lightweight external wall brick mainly used in building envelope not only could reduce the weight of the wall, but also could increase the thermal insulation performance of the wall, and moreover could promote the building energy saving [1]. After a proper amount of pore-forming agents are added to the ceramic raw material, one new type of lightweight external wall brick is made by making the hole by occupying the hole or high temperature foaming during the sintering process. When the temperature is risen to a certain value, pore-foaming agents begin to evaporate and combust until the pore forming agent was almost burnt out and leave blank, leading to the appearance of the closed pores in the body of the ceramic. This is the so-called occupied pore-foaming. High temperature foaming method is that the gas produced by foaming agent of chemical reactions at high temperature is used to prepare porous structure of the lightweight external wall brick.

Many scholars have conducted research on the effect of different pore-forming agents on the properties of sintered products. Ma et al [2-3] investigated the effect of the rice husk and sawdust on physical properties of fly-ash-shale sintered product in different firing systems. Except for the rice husk, sawdust and other fibrous biomass, wheat [4] poppy seeds [5] were used pore-forming agents to prepare porous alumina ceramics. Wu et al [6] prepared diatomite ceramic materials whose volume density, water absorption rate and flexural strength was in sequence $1.05 \mathrm{~g} / \mathrm{cm}^{3}, 51.38 \%$ and 11.29 $\mathrm{MPa}$. Moreover, the diatomite was used as the main raw materials and appropriate plastic clay and fly ash were chosen as supporting materials to make one kind of porous ceramic whose density and flexural strength was in turn 0.7-1.2 $\mathrm{g} / \mathrm{cm}^{3}$ and 7-12 $\mathrm{MPa}$ [7]. There are lots of patents about the porous ceramic by means of high temperature foaming [8-10]. Xu Wei et al [11] sintered the lightweight external wall brick with silicon carbide as the foaming agent in $1200{ }^{\circ} \mathrm{C}$ and the density, water absorption and flexural strength of the bulk was respectively about $1.0 \mathrm{~g} / \mathrm{cm}^{3}, 0.5 \% \sim 1.2 \%$, and $4 \mathrm{MPa} 10 \mathrm{MPa}$. However, only few studies have conducted on the comparative study of both pore-forming agents.

In this study, the lightweight external wall bricks were prepared by means of both pore-forming agents (placeholder pore-forming agent and high temperature foaming agent) to explore the effect of different pore-forming agents on water absorption, density and flexural strength of the lightweight external wall brick. The bran and coal powder were used as placeholder pore-forming agent and SiC was chosen as high temperature foaming agent. 


\section{Experimental}

The preparation of lightweight external wall brick was achieved by using eight steps and three different kinds of pore-forming agent. The steps included batching, ball milling, sieving, staling, granulation, pressing, drying and firing, as shown in Figure 1.

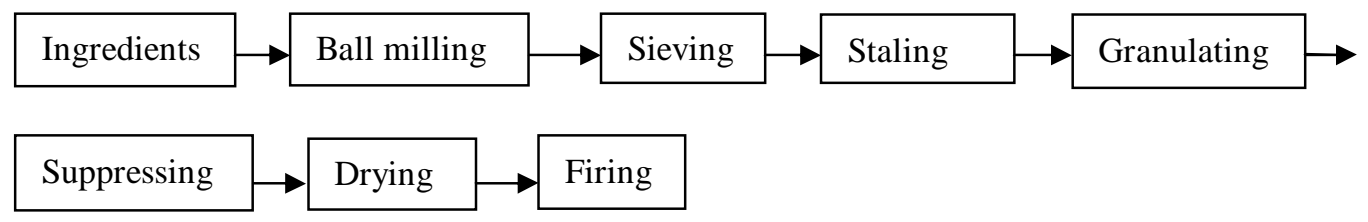

Figure1. Process flow chart of preparation process of lightweight sintering brick

The pressed shaped ceramic body was dried at $105{ }^{\circ} \mathrm{C}$ for $2 \mathrm{~h}$, water content was less than $3 \%$, and sintered in silicon carbon heating furnace with the heating process is $10{ }^{\circ} \mathrm{C} / \mathrm{min}$, retained $1100{ }^{\circ} \mathrm{C}$ for $10 \mathrm{~min}$.

The samples were pressed by the DY-20 electric machine. The water absorption of lightweight external wall brick was measured by vacuum method, the bulk density was measured by Archimedes method, and the bending strength was measured by three point bending method.

\section{Results and discussion}

\section{(1) Effect of rice bran content on the performance of the lightweight external wall brick}

The relationship between the bran content and the water absorption, density and bending strength of the lightweight external wall brick is shown in Figure 1, Figure 2 and Figure 3 respectively. With the increase in content of the pore-forming agent, the water absorption of the lightweight external wall brick exhibited upward trend, while the density and the bending strength decreased continuously. This was because that the bran, as pore-forming agent, was sintered in the body and then forming interlinked pores, resulting in the increase of apparent porosity. The phenomenon could prompt the increase of water absorption rate, leading to the decrease of density and bending strength. Therefore, the lightweight external wall brick made by laterite had problem of high water absorption, and it was difficult to lower the bulks density. In terms of the features of the lightweight external wall brick after sintering, the surface of brick was smooth and had not obvious defects. While the rice bran was added, small holes appeared in the surface of the brick. When the content of bran increased from $10 \mathrm{wt} \%$ to $25 \mathrm{wt} \%$, the defects of exposed holes increased gradually. It was deduced that the greater the content of rice bran was, the more defects were in the surface of lightweight external wall brick.

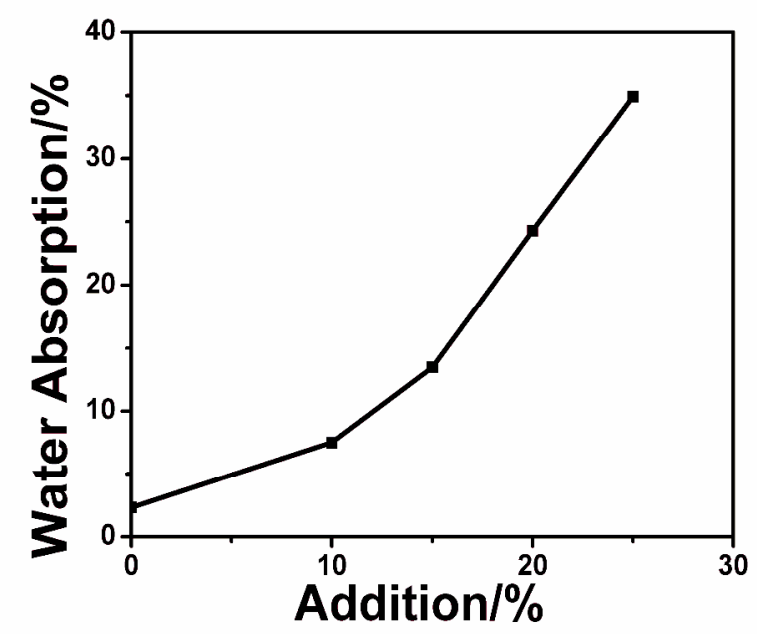

Fig. 1 The dependence of water absorption on bran content

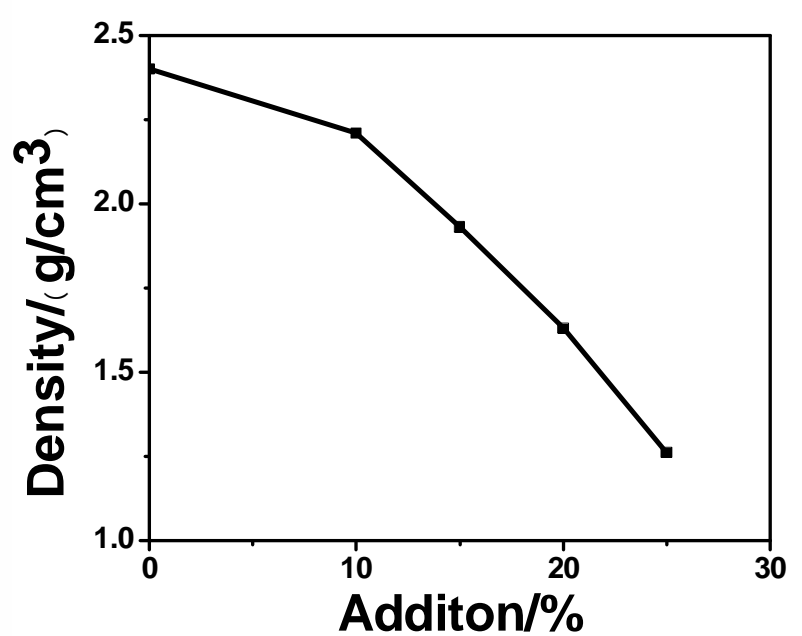

Fig. 2 The dependence of density on bran content 


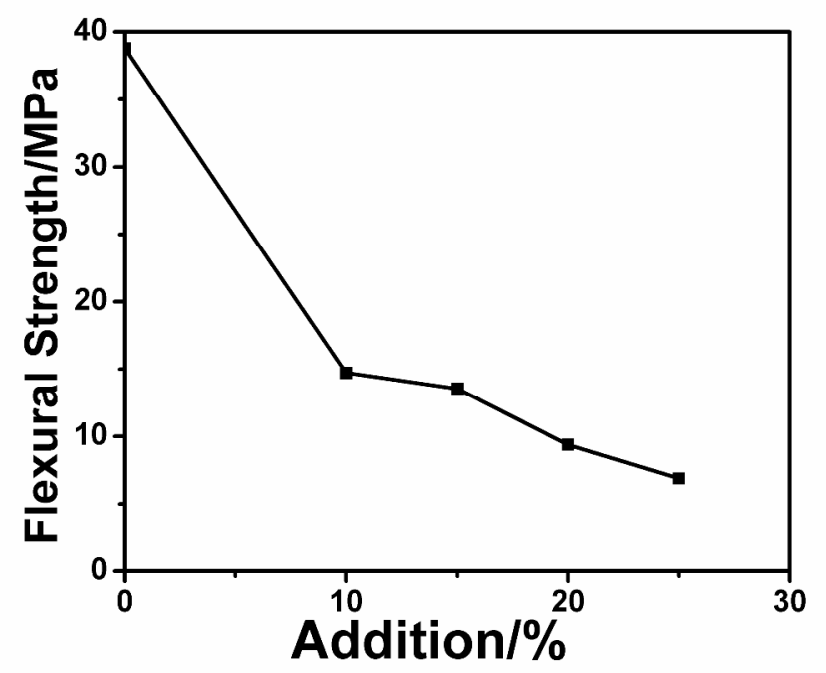

Fig.3 The dependence of flexural strength on bran content

\section{(2) Effect of coal powder content on the performance of the lightweight external wall brick} The dependence of water absorption rate, density and flexural strength on coal powder content is shown in Figure 4, Figure 5 and Figure 6 respectively. With the increase of coal powder content, the water absorption of the lightweight external wall brick increased unremittingly, while the density and the bending strength decreased continuously. Compared with using bran as the pore-forming agents, the density and bending strength of the lightweight external wall brick were not obvious due to the limit of coal powder content. If the coal powder was too much, the reaction would not be completely, and the phenomenon of stratification would be appeared in the body. From the morphology of the lightweight external wall bricks after firing, when coal powder mixing content was more than 10wt\%, the coal powder appeared incomplete oxidation phenomenon in the lightweight external wall brick, and on the surface of the cross-section of the sample, the upper and lower parts were red and black respectively.

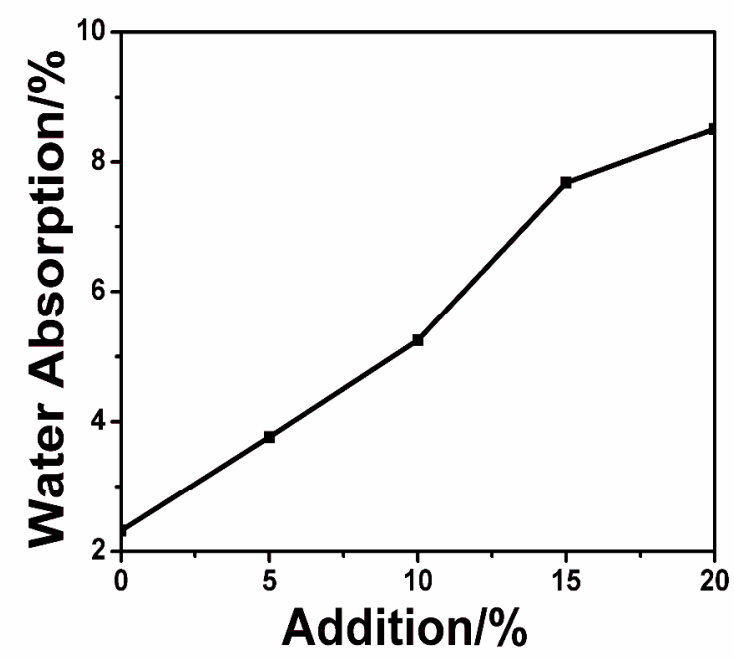

Fig.4 The dependence of water absorption brick on coal powder content

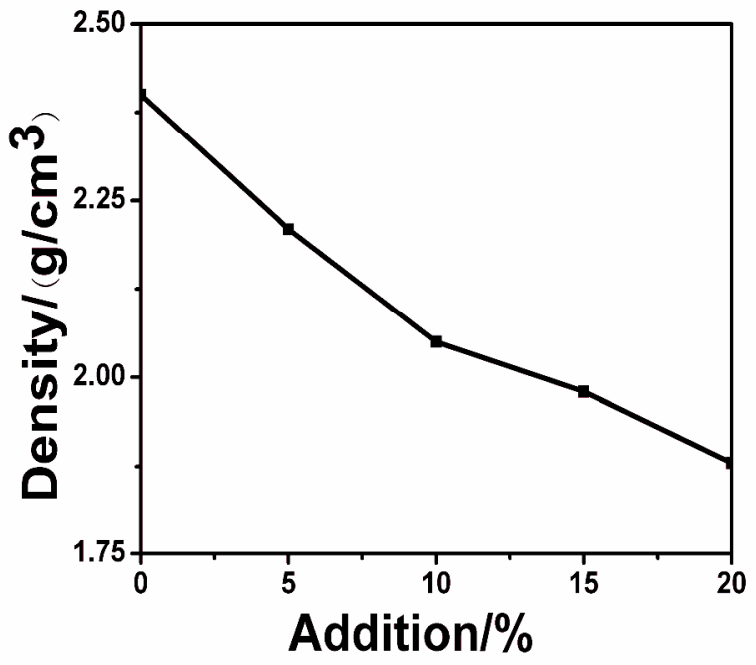

Fig.5 The dependence of density on coal powder content 


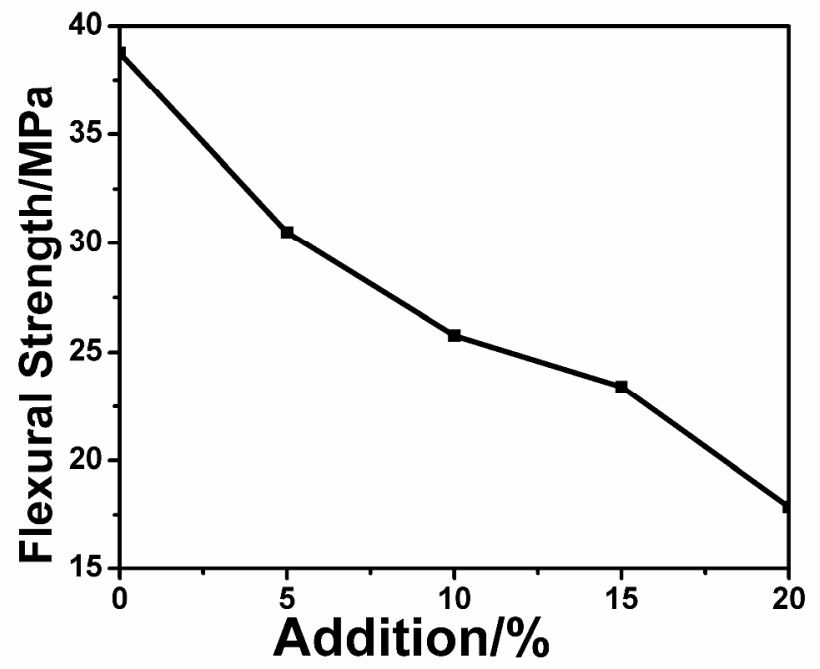

Fig.6 The dependence of flexural strength on coal powder content

\section{(3) Effect of $\mathrm{SiC}$ content on the performance of the lightweight external wall brick}

The relationship between SiC content and lightweight external wall brick density and flexural strength is shown in Figure 7 and Figure 8 respectively. It can be seen that $\mathrm{SiC}$ as high temperature foaming agent was easy to get low density samples, while the strength of as-prepared samples increased. From the morphology of the lightweight external wall bricks after sintering, while the SiC content was less than $0.4 \mathrm{wt} \%$, the morphology of lightweight external wall brick did not change significantly, and the pore points rate was not high and the pore points were not well-distributed. When the SiC content was $0.4 \sim 0.5 \%$, the gas hole in lightweight external wall brick interior showed more uniform.

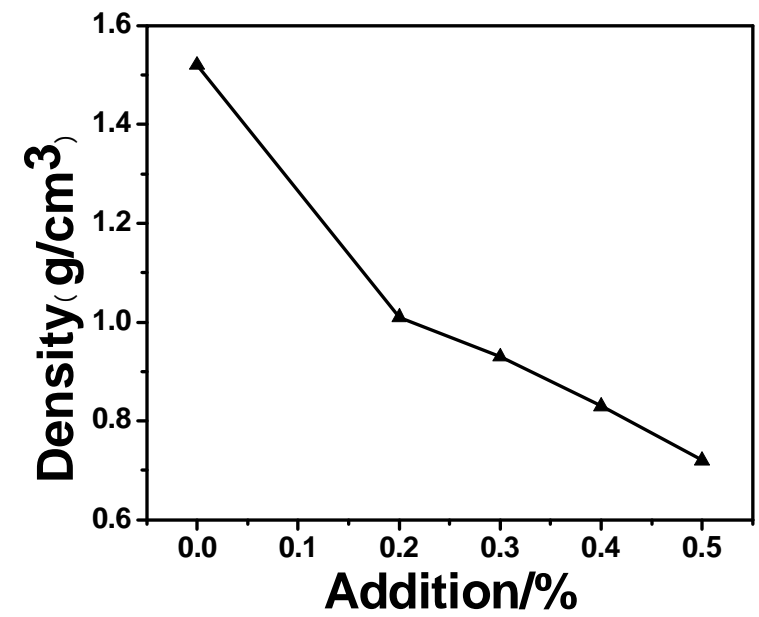

Fig.7 The dependence of density on $\mathrm{SiC}$ content

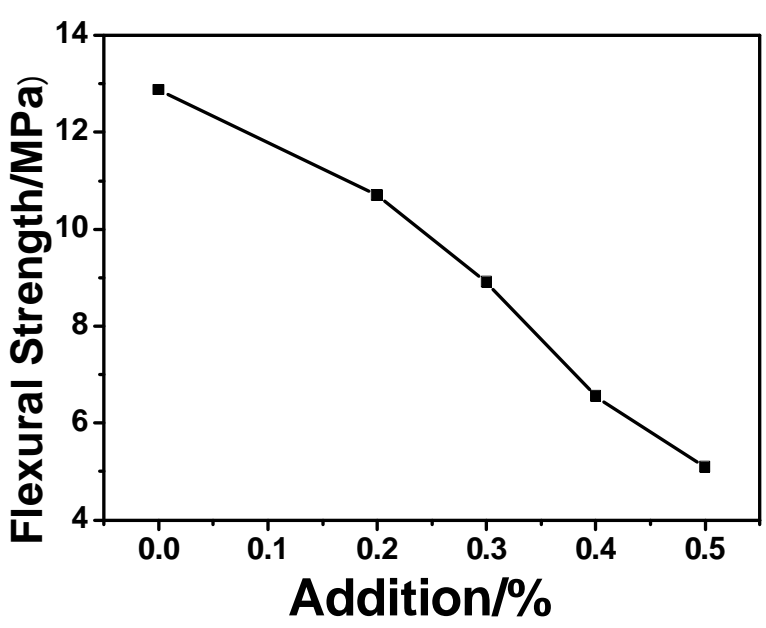

Fig.8 The dependence of flexural strength on $\mathrm{SiC}$ content

With the increase of the added content, $\mathrm{SiC}$ could react with free oxygen in the softening range of ceramic body to produce $\mathrm{CO}$ and $\mathrm{CO}_{2}$, resulting in the appearance of numerous closed pores inside the green body after fired. Hence, the cross-sectional area decreased, leading to the decrease of the stress concentration, and the density and flexural strength. In the meanwhile, a certain amount of glass phase was formed in the surface of the body after sintering. Therefore, its absorption rate was relatively small (generally about $1 \%$ ) [12].

Compared with rice bran and coal powder, $\mathrm{SiC}$ was used as high temperature foaming agent, the surface of lightweight external wall brick was not rough and layered after sintering. The reaction between $\mathrm{SiC}$ and the free oxygen would produce gas, which formed closed pores in the body. On the 
other hand, using bran and coal power as the pore forming agent would not only form a lot of significant porosity in the surface, but also some connected pores in the inner part of body. Therefore, using $\mathrm{SiC}$ as the pore forming agent made the water absorption rate of lightweight external wall brick low; pore porosity of body after sintering was relatively large, and the cross-sectional area of the load pressure decreased which caused the stress concentration, so the flexural strength ratio is small when using bran and coal power as the pore-forming agent.

\section{Conclusion}

In this study, the lightweight external wall brick was prepared by means of dry pressure molding sintering process to explore the effect of the pore-forming agent on its performance. The following conclusions can be drawn:

a) With the increase of the rice bran content, the apparent porosity began to appear on the surface of the lightweight external wall brick and could prompt the increase of water absorption rate, leading to the decrease of density and bending strength.

b) As the content of coal powder increased, its water absorption rate kept the same pace, while the density and compressive strength decreased.

c) With the increase of the $\mathrm{SiC}$ content, $\mathrm{SiC}$ could react with oxygen to generate $\mathrm{CO}$ and $\mathrm{CO}_{2}$, resulting in the appearance of numerous closed pores inside the green body after fired. It was easy to obtain low density lightweight external wall brick which could exhibit better performance.

\section{References}

[1] Hengqing Ye: Ceramics, 1 (1994), p. 26-27. [In Chinese]

[2] Baoguo Ma, Song Mu, Yaocheng Wang, et al: Journal of Functional Materials, 39 (2008), p. 517-520. [In Chinese]

[3] Baoguo Ma, Yaocheng Wang, Song Mu, et al: Journal of Wuhan University of Technology, 4 (2009), p. 38-41. [In Chinese]

[4] K. Prabhakaran, A. Melkeri, N.M. Gokhale, et al: Ceramics International, 33 (2007), p. 77-81.

[5] E. Gregorova, W. Pabst: 33 (2007), p. 1385-1388.

[6] Hongping Wu, Renping Wu, Yan Yu, et al: Bulletin of the Chinese Ceramic Society, 28 (2009), p. 641-645. [In Chinese]

[7] Han Yu, Qing Zhu: Block-Brick-Tile, 5 (1997), p. 32-33. [In Chinese]

[8] Anzhe Rui, Yanchun Liu, Haibin Xia, et al: China. Patent 101186519A. (2008). [In Chinese]

[9] Qi Tang, Bin Lu: China. Patent 101182232A. (2008). [In Chinese]

[10] C. KIM, T. KIM, C.H. KIM, et al: Japan, Patent 2007528331-W. (2007).

[11] Wei. Xu, Shibing Sun, Yingliang Tian, et al: Block-Brick-Tile, 4 (2007), p. 13-15. [In Chinese]

[12] Yading Zhao, Baosheng Zhang, Yong Ge: Journal of Harbin University of Civil Engineering and Architecture, 31 (1998), p. 85-90. [In Chinese] 